\title{
AVALIAÇÃO SENSORIAL DE MAIONESE TRADICIONAL E MAIONESE ENRIQUECIDA COM ERVAS AROMÁTICAS ${ }^{1}$
}

\author{
Jocelem Mastrodi SALGADO ${ }^{2 *}$, Jean Carlos CARRER ${ }^{2}$, Flávia DANIELI ${ }^{2}$
}

\begin{abstract}
RESUMO
O presente trabalho teve como objetivo avaliar a aceitabilidade de amostras de maionese obtidas em laboratório com diferentes tipos de óleos e aromatizadas com ervas. O método de ordenação foi utilizado na avaliação da preferência das amostras produzidas em laboratório e o teste de diferença-do-controle foi aplicado com o intuito de saber se existiam diferenças significativas entre estas. Verificou-se também o nível de aceitação das amostras por escala hedônica de 9 pontos, no qual 28 julgadores avaliaram as amostras pelos atributos qualidade geral do produto, sabor e consistência. De acordo com os resultados obtidos no teste de ordenação, verificouse uma diferença significativa $(\mathrm{p}>0,05)$ quanto à preferência das amostras contendo óleo de gergelim e azeite de oliva e, pelo teste de diferença-do-controle, concluiu-se que as amostras de maionese com os óleos de gergelim, coco e azeite de oliva são diferentes ao nível de significância de $5 \%$, em relação à maionese com óleo de soja (padrão). Na avaliação da aceitação por atributos, verificou-se que não houve diferença significativa de preferência entre as amostras aromatizadas.

Palavras-chave: maionese, óleos vegetais, ervas aromáticas, análise sensorial.
\end{abstract}

\section{SUMMARY}

SENSORY EVALUATION OF TRADITIONAL MAYONNAISE AND ENRICHED MAYONNAISE WITH AROMATIC HERBS. The aim of the present work was to evaluate the acceptance of mayonnaise samples produced in the laboratory containing different oils and aromatized with herbs. The ranking test was used for preference evaluation of the laboratory samples and the difference from the control test was applied with the purpose of finding out if there were significant differences between the samples. The acceptance level of samples by the acceptance test using a hedonic scale of 9 points was also observed, where 28 panelists evaluated the samples by considering their general acceptation, taste and consistency. According to results of the ranking test, a significant difference ( $\mathrm{p}>0.05$ ) was observed regarding the sample's preference containing sesame and olive oil and the difference from the control test can conclude that the mayonnaise samples with sesame, coconut and olive oils are different in the significance level of $5 \%$ with regards to soybean oil mayonnaise. In the attribute test, there was no significant difference of preference between the aromatized samples.

Keywords: mayonnaise, vegetable oils, aromatic herbs, sensory evaluation.

\section{1 - INTRODUÇÃO}

O consumo de alimentos industrializados e o interesse por parte das indústrias na elaboração desses produtos têm aumentado significativamente no Brasil a partir da década de 70. Dentre esses alimentos, uma das melhores opções é a maionese.

Segundo FURLANETTO, LACERDA, CERQUEIRACAMPOS [5], a maionese é uma emulsão composta de óleo, ovos e vinagre, sendo considerada um alimento com alto teor lipídico. De acordo com a legislação brasileira, este produto deve apresentar um mínimo de $65 \mathrm{~g}$ de óleo vegetal comestível/100 g do produto [2].

Os lípides desempenham um papel importante na qualidade de certos produtos alimentares, particularmente em relação às propriedades organolépticas que os tornam desejáveis (sabor, odor, cor, textura). Além destas, conferem valor nutritivo aos alimentos, constituindo uma fonte de energia metabólica, de ácidos graxos essenciais e

\footnotetext{
${ }^{1}$ Recebido para publicação em 18/10/2004. Aceito para publicação em 20/10/2006 (001419)

2Departamento de Agroindústria, Escola Superior de Agricultura Luiz de Queiroz, (ESALQ), Universidade de São Paulo, (USP),

Alimentos e Nutrição - Prédio 3, Av. Pádua Dias, 11, C. P. 9,

CEP 13490-000, Piracicaba, (SP), Brasil

E-mail: jmsalgad@esalq.usp.br

* A quem a correspondência deve ser enviada
}

de vitaminas lipossolúveis [4]. Na maionese, a gordura é essencial ao sabor e textura e sua redução pode afetar a aceitabilidade do produto.

Além disso, um outro fator que pode afetar a qualidade nutricional, segurança, cor, flavor e textura de produtos ricos em óleos comestíveis é o desenvolvimento de rancidez [4, 11]. Este aspecto é de grande importância, não somente sob o enfoque econômico, por perdas devido à diminuição da vida-de-prateleira, mas também pela possibilidade dos radicais livres formados reagirem ou interagirem com outros constituintes dos alimentos, provocando uma queda na qualidade nutricional deles [9].

A proteção dos lípides frente à degradação autoxidativa é garantida pelos antioxidantes. O interesse na pesquisa por antioxidantes naturais tem aumentado nos últimos anos, levando às indústrias farmacêuticas, de alimentos $\mathrm{e}$ cosméticos a terem maior atenção em novas fontes de antioxidantes naturais. Os compostos antioxidantes naturais tem sido isolados de diferentes partes de plantas, tais como sementes, frutas, folhas e raízes [6, 14, 7].

Sendo uma das características dos antioxidantes, retardar o desenvolvimento de sabores e odores desagradáveis ocasionado pela oxidação de ácidos graxos insaturados, usualmente presentes como triacilgliceróis e/ou lípides polares, hoje em dia há uma tendência geral, no processamento de alimentos, de substituir os antioxidantes sintéticos pelos inibidores da 
oxidação natural ou pelo uso preferencial de ingredientes que naturalmente possuem atividade antioxidante [13].

Desde antigamente, especiarias e ervas têm sido usadas não somente para melhorar o sabor e odor em alimentos e estender o tempo de prateleira, mas também pelas suas propriedades anti-sépticas e medicinais [8]. Dentre as mais utilizadas estão o alecrim, orégano, sálvia, etc.

Diante do exposto acima e com a finalidade de aumentar a qualidade nutricional da maionese, o objetivo do presente trabalho foi desenvolver e avaliar o efeito do uso de diferentes tipos de óleos e ervas aromáticas na elaboração de maioneses.

\section{2 - MATERIAL E MÉTODOS}

\section{1 - Material}

Sete amostras de maionese produzidas em laboratório com diferentes tipos de óleo (soja, coco, gergelim e azeite de oliva) e ervas aromáticas (manjericão, manjerona/tomilho e alecrim).

\section{2 - Preparo das amostras}

As amostras foram preparadas e numeradas de acordo com a ordem em que foram obtidas: A (padrão de óleo de soja); B (óleo de coco); C (azeite de oliva); D (óleo de gergelim); E (manjerona/tomilho); F (alecrim); e G (manjericão). A composição das amostras utilizadas é apresentada na Tabela 1.

TABELA 1 - Composição das amostras.

\begin{tabular}{|c|c|c|c|c|c|c|c|}
\hline \multirow[t]{2}{*}{ Componentes } & \multicolumn{7}{|c|}{ Amostras } \\
\hline & A & B & C & D & $\mathbf{E}$ & $\mathbf{F}$ & G \\
\hline Açúcar ${ }^{1}$ & 6 & 6 & 6 & 6 & 6 & 6 & 6 \\
\hline $\mathrm{Sal}^{2}$ & 3 & 3 & 3 & 3 & 3 & 3 & 3 \\
\hline Mostarda $^{3}$ & 3 & 3 & 3 & 3 & 3 & 3 & 3 \\
\hline Gema $^{4}$ & 10 & 10 & 10 & 10 & 10 & 10 & 10 \\
\hline Vinagre $^{5}$ & 13 & 13 & 13 & 13 & 13 & 13 & 13 \\
\hline Óleo soja ${ }^{6}$ & 65 & 32,5 & 16 & 43 & 65 & 65 & 65 \\
\hline Óleo coco ${ }^{7}$ & - & 32,5 & - & - & - & - & - \\
\hline Óleo gergelim ${ }^{8}$ & - & - & - & 22 & - & - & - \\
\hline Azeite de oliva ${ }^{9}$ & - & - & 49 & - & - & - & - \\
\hline Alecrim $^{10}$ & - & - & - & - & - & $\begin{array}{l}1 \mathrm{col} . \\
\text { sopa }\end{array}$ & - \\
\hline Manjerona/tomilho ${ }^{11}$ & - & - & - & - & $\begin{array}{c}1 / 2 \\
\text { col. } \\
\text { sopa } \\
\text { cada }\end{array}$ & - & - \\
\hline Manjericão ${ }^{12}$ & - & - & - & - & - & - & $\begin{array}{l}1 \mathrm{col} . \\
\text { sopa }\end{array}$ \\
\hline
\end{tabular}

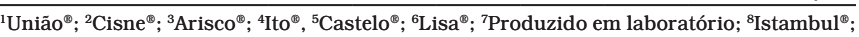
${ }^{9}$ Otoyan $^{\circledast}$; e Kitano ${ }^{\oplus}$.

A quantidade e o tipo de óleo nas amostras B, C e D foram alterados em relação à amostra $\mathrm{A}$, na tentativa de melhorar a qualidade nutricional sem alterar o sabor.

As amostras E, F e G foram aromatizadas com ervas com o intuito de aperfeiçoar seus atributos sensoriais.
Todas as porcentagens foram calculadas sobre a massa total dos ingredientes.

\section{3 - Análise sensorial}

As análises foram realizadas em laboratório de análise sensorial da Universidade. Os testes foram realizados com estudantes universitários e funcionários públicos de ambos os sexos e com idades entre 17 e 50 anos, instruídos para a participação. As amostras foram servidas aleatoriamente aos julgadores em copos plásticos, com quantidades padronizadas ( $10 \mathrm{~g}$ - em função do tipo de amostra) e codificadas com 3 dígitos, obtidos de uma tabela de números aleatórios [10]. Foi fornecida torrada integral e água mineral para limpeza do palato entre a avaliação das amostras [3]. Os dois primeiros testes foram realizados entre 9 e $10 \mathrm{~h}$ e o último entre 15 e $16 \mathrm{~h}$.

Para comparar as amostras de óleo de soja, óleo de coco, azeite de oliva e óleo de gergelim foi utilizado o teste de ordenação.

Para os atributos de aparência, odor e sabor das amostras de óleo de coco, azeite de oliva e óleo de gergelim foi aplicado o teste de comparação múltipla ou diferença-docontrole, preconizado pela ASSOCIAÇÃO BRASILEIRA DE NORMAS TÉCNICAS [1]. As amostras foram apresentadas segundo o delineamento de blocos completos casualizados. Os 26 provadores receberam a amostra padrão (óleo de soja) especificada com a letra P e três amostras codificadas com algarismos aleatórios de três dígitos. Foi solicitado que avaliassem cada uma das amostras-teste em relação à amostra-controle, comparando-as segundo o atributo específico e por meio de uma escala $(0=$ nenhuma diferença de $\mathrm{P}$ a 8 = extremamente diferente de P).

A aceitabilidade das amostras de maionese com manjerona/tomilho, alecrim e manjericão foi avaliada pela escala hedônica. Foram utilizadas três escalas hedônicas: uma para avaliar a aceitação geral dos produtos, uma para avaliar o sabor e outra para avaliar a consistência. Foi solicitado aos 28 provadores que avaliassem as amostras pela escala estruturada de 9 pontos registrando o quanto gostaram ou desgostaram de cada atributo.

Os resultados referentes ao teste de ordenação foram tratados estatisticamente pelo método de Friedman e pela análise de variância. O delineamento experimental utilizado foi o de blocos completos inteiramente casualizados [3, 12]. Os dados obtidos no teste de diferença-do-controle foram avaliados por análise de variância e testes de médias de Dunnett, e os resultados do teste de aceitação foram avaliados por análise de variância e testes de médias de Tukey.

\section{3 - RESULTADOS E DISCUSSÃO}

\section{1 - Avaliação sensorial}

Os resultados da avaliação sensorial no teste de ordenação das amostras de óleo de soja, coco, azeite de oliva e gergelim são mostrados na Tabela 2 . 
TABELA 2 - Avaliação sensorial de preferência de maionese por ordenação para as amostras A, B, C e D.

\begin{tabular}{cc}
\hline Diferença da soma de ordens & Módulos da diferença \\
\hline $\mathrm{Aa}-\mathrm{Ba}$ & $20(\mathrm{~ns})$ \\
$\mathrm{Aa}-\mathrm{Ca}$ & $1(\mathrm{~ns})$ \\
$\mathrm{Aa}-\mathrm{Db}$ & $43(\mathrm{~s})$ \\
$\mathrm{Ba}-\mathrm{Ca}$ & $19(\mathrm{~ns})$ \\
$\mathrm{Ba}-\mathrm{Da}$ & $23(\mathrm{~ns})$ \\
$\mathrm{Ca}-\mathrm{Db}$ & $42(\mathrm{~s})$ \\
\hline
\end{tabular}

(ns) = não significativo; (s) = significativo.

Comparando-se os módulos da diferença com a DMS (diferença mínima significativa), conforme mostrado na Tabela 2, pode-se dizer que houve uma diferença estatística significativa (5\%) entre as amostras de óleo de soja e óleo de gergelim e azeite de oliva e óleo de gergelim, ou seja, a amostra de maionese com óleo de gergelim foi diferente das demais, sendo a menos preferida.

O teste de diferença-do-controle permitiu verificar se as amostras de maionese com diferentes tipos de óleos diferiram significativamente da amostra padrão (óleo de soja) em relação à aparência, odor e sabor.

Os resultados avaliados por análise de variância e testes de médias de Dunnett, demonstraram que todas as amostras diferiram significativamente do controle $(p>0,05)$ em relação a todos os atributos avaliados. Os resultados do teste de diferença-do-controle estão expressos na Tabela 3.

TABELA 3 - Teste de diferença-do-controle das amostras de maionese com diferentes tipos de óleo.

\begin{tabular}{lc}
\hline \multicolumn{1}{c}{ Amostras } & Médias + DP \\
\hline Óleo de soja (Padrão) & $2,57+8,47^{\mathrm{a}}$ \\
Óleo de coco & $6,50+6,86^{\mathrm{b}}$ \\
Azeite de oliva & $6,77+5,56^{\mathrm{c}}$ \\
Óleo de gergelim & $7,42+3,70^{\mathrm{d}}$ \\
\hline
\end{tabular}

Médias com letras iguais na mesma coluna não diferem entre si $(\mathrm{p}<0.05)$.

O teste de aceitação avaliou os atributos de aparência geral do produto, sabor e consistência. Este teste verificou a resposta sensorial dos consumidores ou consumidores potenciais em relação aos produtos obtidos.

Na Tabela 4, são apresentadas às médias dos atributos avaliados no teste de aceitação para as amostras utilizadas nesta etapa do estudo. Observa-se que as notas atribuídas às amostras foram superiores a 6, o que representa na escala hedônica um valor considerável.
Como os maiores valores da escala hedônica equivalem a gostar da amostra, nota-se que, em relação aos atributos aceitação geral do produto, sabor e consistência, a amostra de maionese aromatizada com manjerona/tomilho foi a que obteve maior freqüência do índice hedônico (9), que se refere na escala como "gostei extremamente".

De acordo com os resultados obtidos pela análise de variância, constatou-se que os atributos aspecto geral do produto, consistência e sabor não apresentaram diferença significativa de aceitação ( $\mathrm{p}<0,05)$.

\section{4 - CONCLUSÕES}

- A amostra de maionese aromatizada com manjerona/ tomilho foi a que obteve maior freqüência do índice hedônico (9), que se refere na escala como "gostei extremamente"; $\mathrm{e}$

- Entre as amostras apresentadas, a maionese elaborada com óleo de gergelim obteve a menor aceitação.

\section{5 - REFERÊNCIAS BIBLIOGRÁFICAS}

[1] ABNT. Associação Brasileira de Normas Técnicas. Teste de comparação múltipla em análise sensorial de alimentos e bebidas: NBR 13.526. Rio de Janeiro, 1995, 9p.

[2] BRASIL. Diário Oficial da União. Leis, decretos, etc. Portaria 12/78 da CNPA. Brasília, 1978.

[3] FERREIRA, V. L. P. (Coord.). Análise sensorial - Testes discriminativos e afetivos. Campinas: Sociedade Brasileira de Ciência e Tecnologia de Alimentos, p. 73-77, 2000. (Manual Série Qualidade).

[4] FRANKEL, E. N. Antioxidants in lipid foods and their impact on food quality. Food Chem., Kidlington, v. 57, n. 1, p. 51-55, 1996.

[5] FURLANETTO, S. M. P.; LACERDA, A. A.; CERQUEIRACAMPOS, M. L. Pesquisa de alguns microrganismos em saladas com maionese adquiridas em restaurantes, lanchonetes e "rotisseries". Rev. Saúde Pública., São Paulo, v. 16, n. 6, 1982.

[6] MANCINI-FILHO, J.; VAN-KOIIJ, A.; MANCINI, D. A. P.; COZZOLINO, F. F.; TORRES, R.P. Antioxidant activity of cinnamon (Cinnamomum Zeylanicum, Breyne) extracts. Boll. Chim Farmac., Milano, v. 137, n. 11, p. 443-447, 1998.

[7] MIRANDA, M. S.; SATO, S.; MANCINI-FILHO, J. Antioxidant activity of the microalga Chlorella vulgaris cultered on special conditions. Boll. Chim. Farmac., Milano, v. 140, n. 3, p. 165-168, 2001.

[8] NAKATANI, N. Antioxidants from spices and herbs. In: SHAHIDI, F., ed. Natural antioxidants: chemistry, he-

TABELA 4 - Valores médios obtidos a partir da escala hedônica para as amostras de maionese aromatizadas com manjerona/tomilho, alecrim e manjericão.

\begin{tabular}{lcccccr}
\hline \multicolumn{1}{c}{ Amostra } & $\begin{array}{c}\text { Aceitação geral do } \\
\text { produto }\end{array}$ & Erro padrão & Sabor & Erro padrão & Consistência & Erro padrão \\
\hline Manjerona /tomilho & 7,03 & 4,03 & 7,07 & 4,70 & 7,15 & 2,94 \\
Alecrim & 7,03 & 3,17 & 6,78 & 3,32 & 7,25 & 3,45 \\
Manjericão & 6,60 & 2,59 & 6,57 & 2,59 & 7,42 & 1,73 \\
\hline
\end{tabular}


alth effects and applications. Champaign: AOCS Press, 1997. p. 64-75.

[9] NAWAR, W. W. Lipids. In: FENNEMA, O. R. Food chemistry. 3.ed. New York: Marcel Dekker, 1996. p. 225-319. (Food science and technology).

[10] PEDRERO, F. D. L.; PANGBORN, R. M. 1989. Evaluación Sensorial de los Alimentos - Métodos Analíticos. Alambra Mexicana.

[11] SHAHIDI, F.; WANASUNDARA, P. K. J. P. D. Phenolic antioxidants. Crit. Rev. Food Sci. Nutr., Lauderdale, v. 32, n. 1, p. 67-103, 1992.
[12] SHIROSE, I.; MORI, E. E. M. Estatística aplicada à análise sensorial. Campinas, São Paulo, Ital, 1994.

[13] TSALIKI, E.; LAGOURI, V.; DOXASTAKIS, G. Evaluation of the antioxidant activity of lupin seed flour and derivatives (Lupinus albus ssp. Graecus). Food Chem., Kidlington, v. 65, n. 1, p. 71-75, 1999.

[14] WETTASINGHE, M.; SHAHIDI F. Antioxidant and free radical-scavenging properties of ethanolic extracts of defatted borage (Borago officinalis L.) seeds. Food Chem., Kidlington, v. 67, n. 4, p. 399-414, 1999. 